\title{
Optimising real time clinical librarian support to enhance the evidence base in radiotherapy clinical protocols
}

Carol-Ann Regan, Simon Goldsworthy, and Jessica Pawley

\begin{abstract}
Clinical teams are professionally driven to adopt the latest evidence-based care ensuring optimal outcomes for patients. There can be delays in the latest evidence reaching practice. The radiotherapy multi-professional team in partnership with Clinical Librarians developed a lean process to undertake the real-time evidence-based live update of clinical radiotherapy protocols. Principles of Quality Function Deployment were deployed to create a lean process. The process was evaluated for the percentage difference to the radiotherapy protocol parameters over two years. Satisfaction of the live update was scored from 1: Very dissatisfied to 5: Very satisfied. Since 2014, 12 protocols have been through the process. The live update resulted in $80 \%$ of differences to the clinical protocol compared to the previous two years. Among 10 respondents, a mode of 5 was scored for satisfaction. This novel approach has been successful in providing a lean process ensuring that the latest evidence reaches radiotherapy practice.
\end{abstract}

\section{Authors}

Carol-Ann Regan is retired as Library and Knowledge Services Manager. She was Clinical Librarian at the start of this service development at the Taunton and Somerset NHS Foundation Trust.

Simon Goldsworthy is Principal Research Radiographer in Radiotherapy at the Taunton and Somerset NHS Foundation Trust.

Jessica Pawley is currently Improvement Librarian and was previously Clinical Librarian at the Taunton and Somerset NHS Foundation Trust.

Email: simon.goldsworthy@tst.nhs.uk 


\section{Introduction}

National Health Service (NHS) library specialists are constantly aware of the need to explore ways to ensure their staff meet the needs of the Trust they serve. In 2009, a new cancer centre opened within an acute Trust in the South West of England. The library service took this opportunity to expand their service. At this time there were no Clinical Librarians (CL) operating within the South West. Exploring the evidence for CL models (Winning \& Beverley, 2003), it was decided to develop an embedded CL service to underpin the evidence needs of this newly formed centre.

New teams are professionally driven to adopt the latest practice to ensure the best possible outcome for patients. However, there can be delays in the latest evidence reaching practice for logistical reasons such as resources and time (Goldsworthy, Roe, McGrail, McCormack \& Walther, 2016). The Radiotherapy service within the cancer centre had implemented clinical protocols as the centre opened in 2009; however; these had not been updated systematically with recent evidence in the intervening years. In 2013, the Radiotherapy service was keen to explore a time efficient way to update these and ensure that utilising the skills of the CL the evidence base remained central to frontline delivery.

\section{Background}

The number of CLs within the NHS has grown since 2000 (Harrison \& Beraquet, 2010). A CL can be defined as a professional who works closely with a given clinical team to supply evidence in support of patient care and clinical practice. Winning and Beverley (2003) describes their role "to support clinical decision making and/or education by providing timely, qualityfiltered information to clinicians at the point of need"' (p. 10). Typically, the librarian is still a 
member of the library team with a base in the library. An embedded CL is part of the clinical team with a base giving access to wards, clinicians and patients on a daily basis. They are recognised as part of the clinical team operating on the frontline of clinical delivery.

When we chose to offer a CL service to the new cancer team they were keen that we adopted the embedded approach to enhance visibility and improve healthcare professionals' access to a timely CL service. The vision was to offer training, literature searching and current awareness as the key aspects of the role. However, after preliminary evaluation of the CL role in 2010, it was obvious that the evidence searching skills of the librarian were what was most valued. One clinician fed back that "the clinical library service is crucial to my role ... has been invaluable in providing good peer reviewed and current literature."

By 2013, the role of the CL was fully embedded within the Radiotherapy team. Alongside this there was now a need to efficiently update the clinical radiotherapy protocols with current evidence. With an embedded CL on the team, it was decided their skills could be employed to enable this update to take place and to underpin the protocols with up to date evidence.

Radiotherapy is an effective treatment for many cancers, but must be delivered with millimetre precision to ensure tumour control while avoiding healthy tissue co-irradiation. It is the treatment of choice based on evidence where it is shown to lead to improved clinical outcomes compared to alternative treatments. (Delaney, Jacob, Featherstone \& Barton, 2003). For each anatomical-specific cancer there needs to be a clinical radiotherapy protocol to guide the prescription of radiotherapy. 
Working in partnership, the Principal Radiotherapy Researcher and the CL looked at how evidence based updates of the clinical protocols may be achieved under the auspices of a service development project.

The objectives were to:

1) Use a lean process framework to develop a process for updating radiotherapy protocols assessing multi-professional satisfaction.

2) Evaluate the impact of a CL through measuring the percentage of change to radiotherapy protocols.

\section{Method and methodology}

The prospective service development project was considered a service evaluation by the Oncology service following good clinical practice (Vijayananthan \& Nawawi, 2008). The project was planned through developing a lean methodology advocating the creation of an efficient process flow by eliminating non-value adding activities. (Bonilla, Pawlicki, Perry \& Wesselink, 2008).

A lean process was developed including the principles of Quality Function Deployment (QFD) (Bonilla, Pawlicki, Perry, \& Wesselink, 2008). QFD is a team-based technique that provides a means of identifying and translating customer requirements into technical specifications for product planning, design, process, and production. The term QFD is a loose translation from the Japanese name for this methodology, hin shitsu (quality), ki nou (function), ten kai (deployment) (Guinta \& Praizler, 1993). The methodology consists of a structured procedure that starts with the qualities desired by the customer (Multi-Professional Team), leads through the functions required to provide these services, and identifies the means for deploying 
the available resources to best provide for these services.

Research has found that QFD can provide some short-term benefits such as reducing the cross-functional barriers associated with product development teams and aiding changes in corporate culture. However, over the long-term, QFD has been shown to address the more tangible benefits of reduced cycle time, reduced development cost, and increased productivity (Guinta \& Praizler, 1993). An important benefit of QFD has been its effectiveness in capturing, prioritising and stabilising customer requirements. As with many business practices, the manner in which QFD is implemented will likely have a significant impact on the benefits derived (Griffin, 1992). Team commitment to the methodology is an important success factor (Griffin, 1992).

The lean process was deployed with the following steps (Figure 1):

1) A Multi-Professional Team (MPT) was formed for each update depending on the anatomical cancer site. The team included Therapeutic Radiographers, Oncologists, Radiotherapy Physicists, service leads and CL. The protocols are cancer site-specific and were set to be updated on a regular rotating basis.

2) Prior to each update the MPT provided the CL with search terms. This enabled the CL to conduct a search using a pre-defined list of resources: healthcare databases including the Cochrane database of systematic reviews, point of care tools and guidelines from the main professional and international cancer and healthcare bodies. This list of resources was compiled by the CL in conjunction with the clinical team.

3) The search results were summarised into a hierarchy of evidence (Guyatt et al., 1995) and sent to the MPT before the live update meeting. Any requests for full text papers or 
guidelines could then be actioned ahead of time, thus ensuring the clinicians could review new evidence.

4) The MPT convened for 3 hours to go through the protocol in real-time. The current protocol was displayed on screen and each aspect of the treatment process reviewed in line with evidence provided. A designated person edited the document as it was discussed. The CL also ensured consistent in-text referencing to evidence where changes were made.

5) The CL constructed a sample search strategy and full reference list for inclusion in the finished, updated protocol. This documentation was an important point of reference when there was a change in CL. It ensured a consistency of method.

6) The product of the live update was an evidence-based clinical protocol approved for radiotherapy practice. It was uploaded to the in-house document management system, Q Pulse ${ }^{\circledR}$ for all attendees to approve. 


\section{Live Update process}

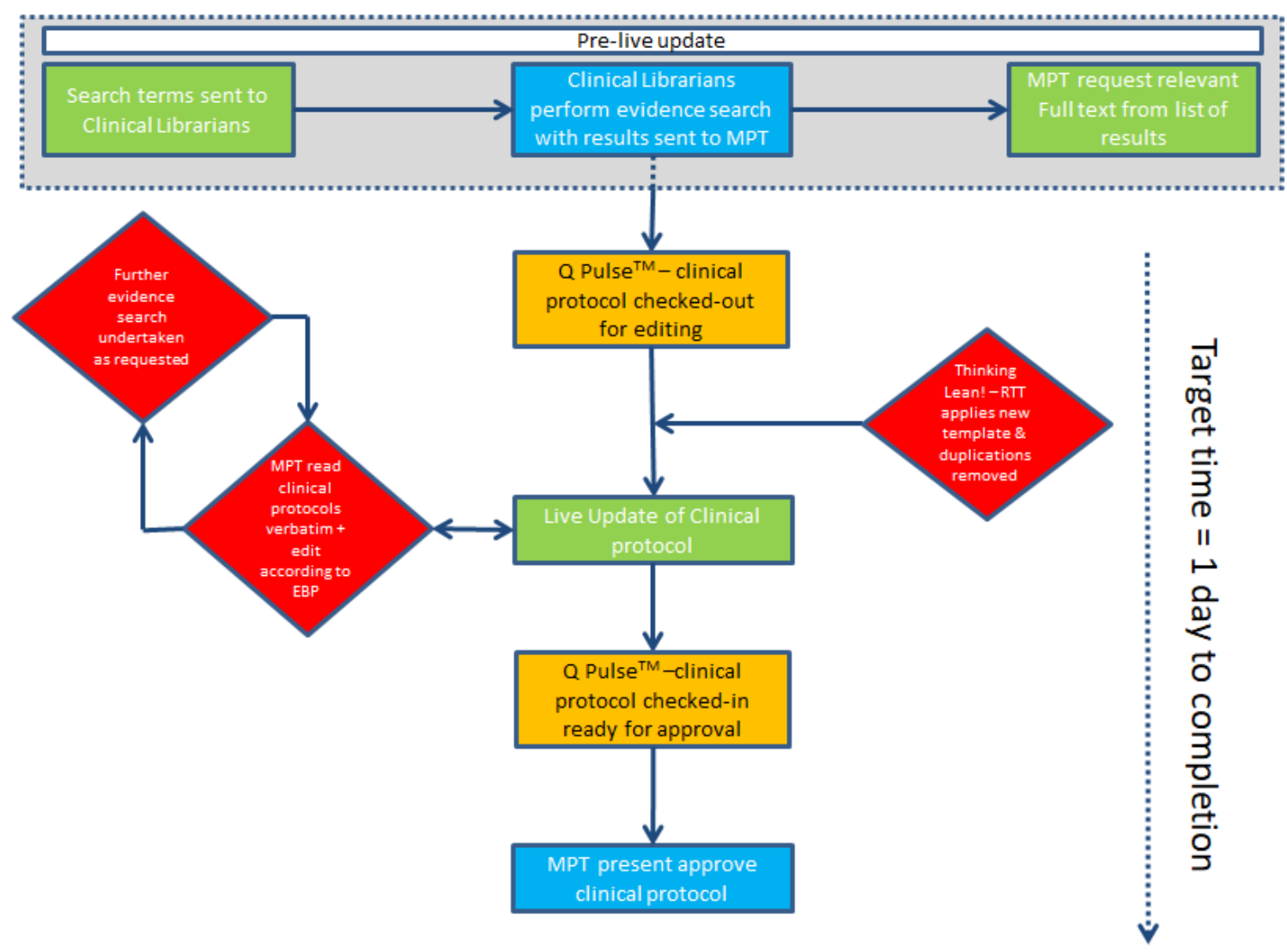

KEY: $\boldsymbol{M P T}=$ Multi-profession team, EBP $=$ Evidence Based Practice, $\boldsymbol{Q}$ Pulse $\boldsymbol{P}^{T M}=$ document management system

Figure 1: Live Update of Clinical Protocols

\section{Data synthesis}

Data synthesis was based on the objectives to assess MPT satisfaction and evaluate the impact of the role of the CL. Satisfaction of the live update was assessed via a short questionnaire and scored on a 5 point Likert scale among the MPT (1 Very dissatisfied; 5 Very satisfied). The mode of response was used to judge overall satisfaction. Questions included: 
- How satisfied were you with the process of the Live Update (e.g. organisation, setting, engagement)?

- How satisfied were you with the end result (e.g. the approved clinical protocol)?

The process of the live update was evaluated for the average percentage difference to the clinical protocol in the following radiotherapy parameters, namely, dose/fractionation, outlining, planning, image guidance and treatment delivery compared to the previous 2 years. The initial evaluation was by the Principal Radiotherapy Researcher with a random selection independently reviewed by the CL.

\section{Results}

In terms of satisfaction from the MPT, among 10 respondents, a mode of 5 (range $=2-5$ ) was scored for how satisfied they were with the process and end result (Figures 2-3). Free text feedback was also elicited. Comments included:

- "A novel process which really improved MPT working"

- "Having the CL involved is impressive and ensures evidence based practice" 
Figure 2: How satisfied were you with the process of the Live Update (e.g. organisation, setting, engagement)?/

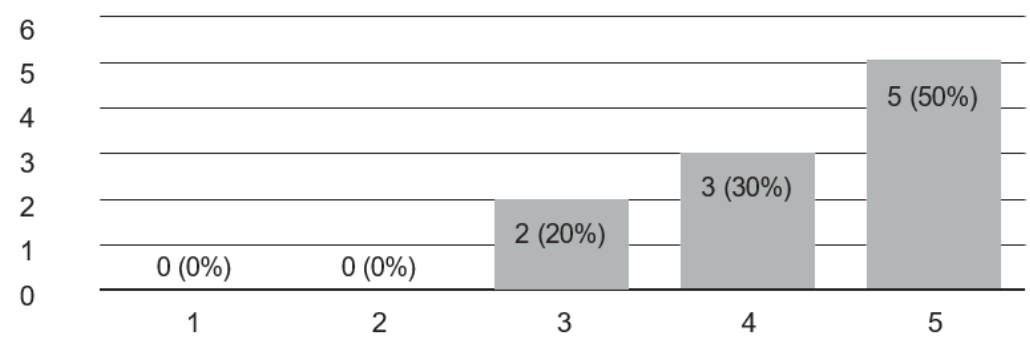

Figure 3: How satisfied were you with the end result (the approved clinical protocol)?

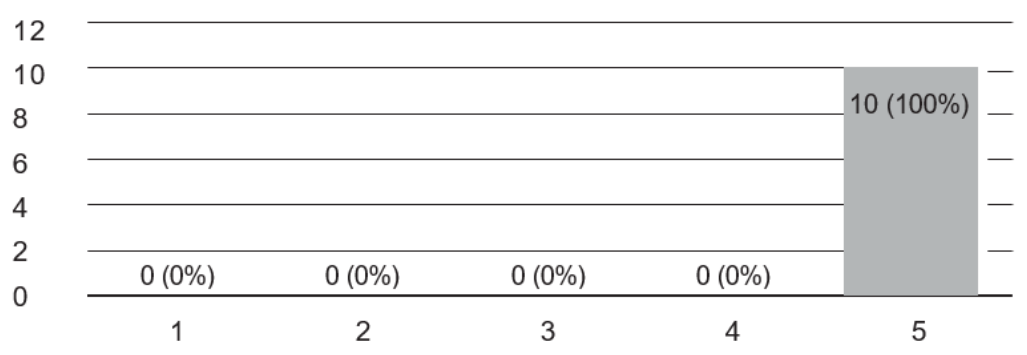

We measured the changes to the radiotherapy perimeters to evaluate the impact of the CL. The live updates resulted in a median difference of $80 \%$ in the radiotherapy parameters (range $40 \%$ to $100 \%$ ) to the clinical protocols compared to the previous two years before the process (Table 1). From 2014-2018, 12 protocols have been through the process, with one having been through a second time. 


\begin{tabular}{|c|c|c|c|}
\hline SITE & Year 1 to year 2 - differences (yes/no) & Year 2 to live update - differences (yes/no) & $\%$ Difference \\
\hline \multicolumn{4}{|c|}{ 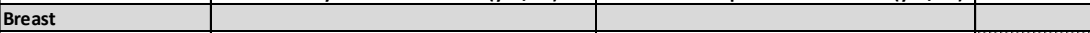 } \\
\hline Dose/\# & no & yes & \\
\hline Outlining & no & yes & \\
\hline Planning & yes & yes & \\
\hline IGRT & no & yes & \\
\hline Treatment delivery & no & yes & \\
\hline Total $=$ & 1 & 5 & $80 \%$ \\
\hline \multicolumn{4}{|l|}{ Lung } \\
\hline Dose/\# & no & yes & \\
\hline Outlining & no & yes & \\
\hline Planning & no & yes & \\
\hline IGRT & no & yes & \\
\hline Treatment delivery & no & yes & \\
\hline Total $=$ & 0 & 5 & $100 \%$ \\
\hline \multicolumn{4}{|l|}{ Oesophagus } \\
\hline Dose/\# & no & yes & \\
\hline Outlining & no & yes & \\
\hline Planning & no & yes & \\
\hline IGRT & no & yes & \\
\hline Treatment delivery & no & No & \\
\hline Total $=$ & 0 & 4 & $80 \%$ \\
\hline \multicolumn{4}{|c|}{ Upper Gastrointestinal } \\
\hline Dose/\# & no & no & \\
\hline Outlining & no & no & \\
\hline Planning & no & Yes & \\
\hline IGRT & no & Yes & \\
\hline Treatment delivery & no & Yes & \\
\hline Total $=$ & 0 & 3 & $60 \%$ \\
\hline \multicolumn{4}{|l|}{ Prostate } \\
\hline Dose/\# & Yes & Yes & \\
\hline Outlining & Yes & Yes & \\
\hline Planning & Yes & Yes & \\
\hline IGRT & No & Yes & \\
\hline Treatment delivery & No & Yes & \\
\hline Total $=$ & 3 & 5 & $40 \%$ \\
\hline \multicolumn{4}{|l|}{ Bladder } \\
\hline Dose/\# & no & Yes & \\
\hline Outlining & no & Yes & \\
\hline Planning & no & Yes & \\
\hline IGRT & no & Yes & \\
\hline Treatment delivery & no & Yes & \\
\hline Total $=$ & 0 & 5 & $100 \%$ \\
\hline \multicolumn{4}{|l|}{ Head \& neck } \\
\hline Dose/\# & no & Yes & \\
\hline Outlining & no & Yes & \\
\hline Planning & Yes & Yes & \\
\hline IGRT & no & Yes & \\
\hline Treatment delivery & no & Yes & \\
\hline Total $=$ & 1 & 5 & $80 \%$ \\
\hline \multicolumn{4}{|l|}{ Haematology } \\
\hline Dose/\# & no & Yes & \\
\hline Outlining & no & Yes & \\
\hline Planning & no & Yes & \\
\hline IGRT & no & Yes & \\
\hline Treatment delivery & no & Yes & \\
\hline Total $=$ & 0 & 5 & $100 \%$ \\
\hline \multicolumn{4}{|l|}{ Palliative } \\
\hline Dose/\# & no & Yes & \\
\hline Outlining & no & Yes & \\
\hline Planning & no & Yes & \\
\hline IGRT & no & Yes & \\
\hline Treatment delivery & no & Yes & \\
\hline Total $=$ & 0 & 5 & $100 \%$ \\
\hline Median & & & $80 \%$ \\
\hline
\end{tabular}

Table 1. \% change to clinical protocols between standard update and live update 


\section{Discussion}

The objectives of this service development were to use a lean process framework to develop a process for updating radiotherapy protocols assessing multi-professional satisfaction and to evaluate the impact of a CL through measuring the percentage of change to radiotherapy protocols. Evidence from the literature on QFD suggested that there would be a reduction in time cycles and increased productivity. The process developed by the Principal Radiotherapy Researcher and CL enabled a protocol to be updated in a few hours rather than over a protracted period. It saved valuable clinician time, improved team working and embedded current evidence into practice.

To assess the impact of the process and end results we measured MPT satisfaction (mode $=5$, range $3-5 \&$ mode $=5$ range 0 ) and evaluated the changes made to the radiotherapy parameters. These findings suggested that most of the MPT were satisfied with the process and end results. A few lower scores for satisfaction for the process demonstrate that further refinements should be explored. The live updates resulted in $80 \%$ difference to the radiotherapy parameters which in clinical practice means a change to a patient's treatment prescription or how the radiotherapy is delivered to directly improve patient outcomes. The update process has fostered good working relationships and ensured clear documentation of the literature review process, search strategy, pre-defined list of resources and referencing. The process has been sustainable and replicable even with changes in both MPT and the role of CL.

There have been a few limitations arising within the process which could have resulted in a lower satisfaction score amongst some of the MPT. On occasion it has not been possible for the CL to attend the live update due to other service demands. However, the CL continued to support 
the process by providing the literature search for evidence ahead of time. One noticeable difference found from non-attendance is that clinicians were less likely to follow up with the CL after the meeting, due to the demands of their own roles. In these instances, the CL has had to be proactive in approaching the clinicians to complete any outstanding issues or requests for evidence left over from the update meeting. This ensured the consistency of the process, and underpinned the importance of evidence to support the update. One issue around any follow up searches that may arise from the live update was that due to the busyness of clinicians there was not always a timely response to any queries posed by the CL. Although the findings of this service development are not generalisable, we recommend that clinical services adopt the process of live updates through a process of evaluation.

CLs support the clinical teams in which they work with evidence to inform practice and ultimately patient care. This initiative to update radiotherapy clinical protocols in a time efficient way underpinned by evidence is to our knowledge the first known project to integrate CL skills with clinical services using a lean QFD methodology to influence real-time updates.

\section{Conclusion}

The novel approach of the live update has been successful in providing a lean process and ensuring that the latest evidence reaches clinical Radiotherapy practice. An innovative collaboration incorporated CLs within the RT MPT for ensuring an efficient, methodical approach and improved team working. The real-time live update resulted in a small investment of time from the MPT, but ensured up to date clinical practice based on the latest evidence and improved patient outcomes. Whilst the lean method process has only been used in one department within our Trust, we very much feel that it would be worth replicating to further test 
its impact. Due to positive patient outcomes we do recommend that other CL services also trial this approach with relevant teams. This is definitely a role CLs can play in directly contributing to patient care but more importantly ensuring that protocol updates are time efficient and evidence-based.

\section{References}

Bonilla, C., Pawlicki, T., Perry, L., \& Wesselink, B. (2008). Radiation oncology lean Six Sigma project selection based on patient and staff input into a modified quality function deployment. International Journal of Six Sigma and Competitive Advantage, 4(3), 196-208.

Delaney, G., Jacob, S., Featherstone, C., \& Barton, M. (2003). Radiotherapy in cancer care: Estimating the Optimal Utilisation from a review of evidence-based clinical guidelines (p. 21). Liverpool BC: Collaboration for Cancer Outcomes Research and Evaluation (CCORE). Retrieved from https://canceraustralia.gov.au/sites/default/files/publications/radiotherapyreport1_504af01e8daf0.pdf

Goldsworthy, S., Roe. B., McGrail, S., McCormack, S., \& Walther, J. (2016). Developing and implementing a Radiotherapy Research Activity Assessment Tool (RAAT): A prospective feasibility study. Journal of Radiotherapy in Practice, (15)3, 223-231.

Griffin, A. (1992). Evaluating QFD's use in US firms as a process for developing products. Journal of Product Innovation Management, 9(3), 171-187.

Guinta, L. R., \& Praizler, N. C. (1993). The QFD book: The team approach to solving problems and satisfying customers through Quality Function Deployment. New York: AMACOM Books. 
Guyatt, G. H., Sackett, D. L., Sinclair, J. C., Hayward, R., Cook, D. J., \& Cook, R. J. (1995). Users' guides to the medical literature. IX. A method for grading health care recommendations. Evidence-Based Medicine Working Group. Journal of the American Medical Association, 274(22), 1800-1804.

Harrison, J., \& Beraquet, V. (2010). Clinical librarians, a new tribe in the UK: Roles and responsibilities. Health Information and Libraries Journal, 27(2), 123-132.

Vernooij, R. W., Sanabria, A. J., Solà, I., Alonso-Coello, P., \& Martínez García, L. (2014). Guidance for updating clinical practice guidelines: A systematic review of methodological handbooks. Implementation Science, 9(1). Retrieved from https://implementationscience.biomedcentral.com/articles/10.1186/1748-5908-9-3

Vijayananthan A., \& Nawawi, O. (2008). The importance of good clinical practice guidelines and its role in clinical trials. Biomedical Imaging and Intervention Journal, 4(1), e5. Retrieved from https://www.ncbi.nlm.nih.gov/pmc/articles/PMC3097692/

Winning, M. A., \& Beverley, C. A. (2003). Clinical librarianship: A systematic review of the literature. Health Information and Libraries Journal, 20 (s1), 10-21. 